Les paysans peuvent-ils devenir banquiers? Épargne et crédit en Afrique, D. Gentil et Y. Fournier, 1993, Syros, Dijon.

\title{
Elsa Faugère
}

\section{(2) OpenEdition Journals}

Édition électronique

URL : http://journals.openedition.org/apad/2703

DOI : $10.4000 /$ apad.2703

ISSN : 1950-6929

Éditeur

LIT Verlag

Édition imprimée

Date de publication : 1 décembre 1993

Référence électronique

Elsa Faugère, «Les paysans peuvent-ils devenir banquiers? Épargne et crédit en Afrique, D. Gentil et Y. Fournier, 1993, Syros, Dijon. », Bulletin de l'APAD [En ligne], 6 | 1993, mis en ligne le 10 mars 2008, consulté le 23 septembre 2020. URL : http://journals.openedition.org/apad/2703 ; DOI : https:// doi.org/10.4000/apad.2703

Ce document a été généré automatiquement le 23 septembre 2020.

Bulletin de I'APAD 


\section{Les paysans peuvent-ils devenir banquiers? Épargne et crédit en Afrique, D. Gentil et Y. Fournier, 1993, Syros, Dijon.}

\section{Elsa Faugère}

1 D. Gentil et Y. Fournier, spécialistes du crédit à l'IRAM (Institut de Recherche et d'Application de Méthodes de développement rural), analysent dans cet ouvrage les différentes formes d'épargne et de crédit, en Afrique de l'Ouest principalement. Leur méthode d'analyse se caractérise par les cinq points suivants :

- une mise en perspective historique des institutions financières

- l'utilisation de connaissances pluri-disciplinaires (financières, économiques, politiques, anthropologiques...)

- l'appui sur des exemples très localisés

- le comparatisme géographique

- des propositions concrètes tirées de ces observations empiriques, propositions qui prônent la nécessaire participation des populations à la mise en œuvre et au fonctionnement des systèmes d'épargne-crédit.

2 L'histoire des mécanismes d'épargne-crédit qui constitue la première partie du livre, commence en Afrique de l'Ouest, dans les années 1925-1930 (pour le secteur officiel), par des tentatives de transfert de modèles occidentaux, Crédit Agricole et Sociétés Indigènes de Prévoyance. Sous-tendues par deux logiques différentes, respectivement une logique bancaire et une logique du développement, ces systèmes d'épargne-crédit se soldent par des échecs dus à la mise à l'écart des paysans. Ces derniers tentent en effet de tirer le maximum de bénéfices et d'utiliser toutes les failles de ces modèles exogènes.

Tirant parti de ces échecs, des banquiers ont cherché dans les années 1960 à trouver des solutions plus adaptées aux spécificités locales en faisant jouer des garanties morales, la caution solidaire. Le rapide succès de ces expériences mutualistes et leur 
extension conduisirent à un relâchement dans l'encadrement et dans la diffusion des informations ainsi qu'à une baisse de motivation de la part des populations. Les mauvais payeurs n'étant pas sanctionnés, une solidarité dans le non remboursement se propage, entraînant l'échec du système. A ces facteurs internes s'ajoutèrent des contraintes externes (détérioration des rapports de prix, malaise politique, aléas climatiques...) qui contribuèrent à l'échec des expériences mutualistes.

4 À partir des années 1970-1980, les modes de financement se diversifient (Banques de Développement, COOPEC, ONG...) et entrent souvent en concurrence, chacun préconisant un modèle particulier: commencer par l'épargne ou par le crédit, privilégier les remboursements ou s'attacher à la diffusion des innovations techniques... Chaque institution financière présente des avantages et des inconvénients que les auteurs décrivent dans leurs grandes lignes avant de procéder dans la deuxième partie, à une analyse minutieuse de quatre d'entre elles, représentatives des quatre secteurs qui structurent le champ financier: le secteur "autonome" (informel), le secteur intermédiaire, le secteur des projets de développement et le secteur bancaire. Tous les mécanismes d'épargne-crédit peuvent se classer dans l'un de ces secteurs qui se caractérisent par le degré de participation des bénéficiaires et par la priorité donnée aux nonnes bancaires ou aux nonnes de développement.

5 À travers des exemples localisés et divers géographiquement (Afrique, Asie et Amérique $\mathrm{du}$ Sud), les auteurs mettent en évidence les causes de leurs réussites ou échecs, prenant toujours en compte les spécificités locales et les contraintes macro (sociologiques, économiques, politiques). La reconnaissance de la diversité des stratégies des acteurs en présence, tant du côté des "développeurs" que du côté des "développés", ainsi que des contraintes qui pèsent sur eux, tant au niveau micro que macro, permet aux auteurs, de mettre en évidence les spécificités de chaque situation et leurs points communs et d'en tirer des enseignements. A partir de là ils font, dans la troisième partie, des propositions pour améliorer les pratiques des acteurs impliqués dans le champ financier.

6 La méthode qu'ils proposent pour améliorer les systèmes d'épargne-crédit, c'est-à-dire pour que l'offre financière corresponde à la demande dans chaque cas particulier, fait appel à un des outils des sciences sociales: le recueil de discours par des entretiens, discussions ou questionnaires. C'est à partir de ces discours qu'ils appellent leur matériau de base qu'ils proposent de construire leurs hypothèses d'action. Si la prise en compte des dynamiques sociales et des spécificités culturelles sont tout à fait indispensables à toute action de développement et, dans le cas présent, à la mise en place ou à l'amélioration des formes d'épargne-crédit, il semble que la méthode proposée par D. Gentil et Y. Fournier, à savoir le recueil et l'analyse des discours des acteurs concernés, ne peut suffire à comprendre la complexité des stratégies et logiques en présence. L'observation des conduites effectives des acteurs doit être effectuée en même temps que le recueil de leurs discours, par des professionnels des sciences sociales, sociologues ou anthropologues.

7 Cette nécessaire collaboration implique une adaptation des méthodes utilisées par ces derniers afin de fournir aux praticiens du développement, dans des délais suffisamment brefs, des connaissances socio-anthropologiques utilisables. 


\section{AUTEUR}

\section{ELSA FAUGÈRE}

École des Hautes Études en Sciences Sociales, Marseille 